\title{
Additional thoughts on the new treatment Kybella
}

\author{
Joel L Cohen, MD,${ }^{1-3}$ David LK Chen, MD,${ }^{4}$ Jeremy B Green, MD, ${ }^{5,6}$ and John H Joseph, MD ${ }^{7-9}$
}

\section{Abstract}

With the recent approval of Kythera Biopharmaceuticals (Westlake Village, California) submental fat injectable treatment Kybella, there comes a lot of excitement-but, also a bit of concern. The idea of having a nonsurgical, nonliposuction treatment for people who have a double chin is certainly exciting. The hope has been that this new treatment will provide opportunities for the many people out there who are focused on the appearance of their submental fullness to have access to a safe, affordable, and reliable treatment. With the approval of this product, there are several misconceptions that the authors herein discuss.

Semin Cutan Med Surg 34:138-139 () 2015 Frontline Medical Communications

$\mathrm{K}$ ybella (Kythera Biopharmaceuticals, Westlake Village, California) is a first-in-class injectable agent approved for the treatment of submental fullness. It is a proprietary formulation of synthetic (nonanimal, nonhuman) deoxycholate that has gone through a decade of development and rigorous clinical trials, including trials that document significant improvement that is correlated with objective improvement on magnetic resonance imaging. ${ }^{1,2}$ It has been found that this deoxycholate functions to dissolve fat through a nonspecific cell lysis mechanism. ${ }^{3}$ There are some misconceptions that are already becoming clear as a result of the media's portrayal of the approval and launch of this product.

\section{Misconceptions}

Kybella is without downtime or potential side effects?

Although Kybella is an injectable and a less invasive treatment than surgery or liposuction, it is not without side effects. In the US

${ }^{1}$ Department of Dermatology, University of Colorado, Aurora.

${ }^{2}$ AboutSkin Dermatology and DermSurgery, Englewood and Lone Tree, Colorado.

${ }^{3}$ Department of Dermatology, University of California at Irvine.

${ }^{4}$ Department of Dermatology, University of Colorado, Aurora.

${ }^{5}$ Skin Associates of South Florida, Coral Gables.

${ }^{6}$ Department of Dermatology \& Cutaneous Surgery, University of Miami, Florida.

${ }^{7}$ Director, John H Joseph MD, A Medical Corporation, Beverly Hills, California.

${ }^{8}$ Director, Clinical Testing of Beverly Hills, California.

${ }^{9}$ Assistant Clinical Professor, University of California, Los Angeles. Disclosures: Dr Chen has nothing to disclose. Drs Cohen and Green have served as investigators for Kythera and Neothetics. Dr Cohen is a consultant for Kythera. Dr Joseph is a former shareholder of Kythera Biopharmaceuticals and a clinical trial investigator outside of the submitted work.

Correspondence: Joel L Cohen, MD; 499 E Hampden Ave \#450, Englewood, CO 80113. Email: jcohenderm@yahoo.com.
Food and Drug Administration (FDA) study, the patients receiving the active drug had the following side effects: edema/swelling (87\%), hematoma/bruising (72\%), pain (70\%), numbness $(66 \%)$, erythema $(27 \%)$, induration $(23 \%)$, and temporary marginal mandibular nerve dysfunction resulting in an asymmetric smile (4\%). ${ }^{1,4}$ In the placebo group, bruising at $70 \%$ was the only comparable adverse event to the active drug Kybella. ${ }^{4}$

\section{Kybella is a one-time treatment?}

Kybella is not a "one and done," single treatment cure for the double chin, but rather requires a series of injections spaced at least a month apart-with most patients having 3-5 injection sessions (average 4.5 sessions). This average of 4.5 treatments may likely be falsely elevated due to the nature of clinical trials to treat patients to apparent full correction, and thus continuing the injection sessions for those who may still have some small degree of persistent submental fat. The reality of upcoming clinical practice with this product may be that patients would be content with the improvement seen at an earlier time point after fewer treatments. In addition, the difference of treating to maximal improvement in a study "because we can," versus treating a patient who is paying for each injection may result in less "real world" treatment sessions than seen in the FDA pivotal trial.

\section{Kybella can replace a neck lift or other neck rejuvenation procedures?}

Kybella treatment is meant specifically for patients that have excess submental fat - which can be pinched and confirmed to be preplatysmal - and not for patients that have other neck conditions without prominent submental fat, such as significant skin laxity to the area. In fact, in some patients treated with Kybella, there may be an unmasking of this hidden laxity or even exposure of prominent platysmal muscle bands with the sequential Kybella injections over time. Tightening devices (such as ultrasound or radiofrequency) or even surgery may then be considered to treat this laxity. For newly revealed or unmasked prominent platysmal bands, botulinum toxin or surgery may be considered as well. Some patients who received ATX-101 (previous name for Kybella) had improvement in neck skin laxity, but this is not the case for all patients.

\section{The effects of Kybella are permanent?}

The effects of this treatment are very likely to last a few years and may even possibly be permanent, especially in the absence of significant weight gain; however, we don't yet know how long the treatment will last. The approval of Kybella and worldwide studies to date (including studies in the United States) show that responders can have a durable effect of treatment up to 12 weeks after the 
last treatment, but it may be longer; and we are awaiting data from long term follow-up studies. ${ }^{1,4,5}$

\section{Potential concerns Off-label uses}

Although Kybella has been FDA approved for the treatment of submental fullness, it will most certainly be injected into unstudied and unapproved areas off-label. Patients, physicians, and media are already discussing using Kybella to correct central abdominal fat, the "muffin top" or "love handle" abdominal fat overhang, lipomas, buttock fat, thigh fat, upper-arm fat, cellulite, and infraorbital fat pads. Off-label use of other cosmetic injectable agents such as botulinum toxin and filler agents are already a reality in clinical practice, and we fully expect this to be the case with Kybella. Nonetheless, using Kybella in areas other than the approved submental fat pad region could potentially be effective; and, eventually, some of these regions will likely be studied through investigator-initiated pilot projects and subsequent large-scale studies. This means that right now, we simply do not know how Kybella will affect these areas, how much dose may be needed, what type of side-effects to anticipate, and how to avoid potential pitfalls. We do not yet know if different types of fat in different areas will behave differently with the same injected medication.

\section{Compounded deoxycholic acid}

As mentioned previously, Kybella itself is a purified, laboratorysynthesized nonanimal and nonhuman deoxycholate that has gone through almost a decade of development and rigorous welldesigned FDA studies. Historically, FDA-nonapproved formulations of deoxycholic acid has been compounded (especially with phosphatidylcholine) for fat reduction mesotherapy. ${ }^{6}$ Without significant oversight, compounded agents are clearly not standardized between different pharmacies, or even sometimes between different batches from the same pharmacy — as has been documented with topical anesthetics. ${ }^{7,8}$ Furthermore, there is little, if any, data to support the safety and efficacy of these compounded agents. Nonetheless, with the relatively high price-point of the FDA-approved Kybella product, we have concerns that unproven, compounded deoxycholate products may once again flood the market.

\section{Undertreatment as a cost-saving practice}

Another concern of Kybella we foresee is the practice of intentional undertreatment to keep practice costs low. With undertreatment (eg, using only $2 \mathrm{~mL}$ of product when the number of dots on the treatment grid may indicate $4 \mathrm{~mL}$ is needed), patients may spread the word that Kybella "didn't work for them" when in reality they did not receive adequate dosing.

\section{Inadequate Kybella training}

As there are few designated Kybella trainers and training programs are in the formation stages, there is a potential for significant risks because of a lack of supervision or insufficient training. For instance, erroneous injection of Kybella into a "no-fly zone," like the jowls, can risk marginal mandibular nerve dysfunction. Like with any treatment, and especially cosmetic treatments, patients need to take some responsibility to know the qualifications and training of their physician injectors.

\section{Conclusion}

Physicians and patients are interested in the use of Kybella for minimally invasive fat contouring. However, there remain misconceptions in the media and general population regarding its side-effect profile and the durability of Kybella. Furthermore, while Kybella may have potential use beyond its FDA-approved submental indication, off-label attempts should be fully explained to the patient. We urge physicians to avoid the use of unproven compounded deoxycholate "substitutes," to follow the recommended dosing regimen of Kybella, and to become familiar with anatomy and adverse events associated with Kybella to achieve optimal outcome and patient satisfaction.

\section{References}

1. Dayan SH, Jones DH, Carruthers J, et al. A pooled analysis of the safety and efficacy results of the multicenter, double-blind, randomized, placebo-controlled phase 3 REFINE-1 and REFINE-2 trials of ATX-101, a submental contouring injectable drug for the reduction of submental fat. Plast Reconstr Surg. 2014;134(4S-1):123.

2. McDiarmid J, Ruiz JB, Lee D, Lippert S, Hartisch C, Havlickova B. Results from a pooled analysis of two European, randomized, placebo-controlled, phase 3 studies of ATX-101 for the pharmacologic reduction of excess submental fat. Aesthetic Plast Surg. 2014;38(5):849-860.

3. Rotunda AM, Suzuki H, Moy RL, Kolodney MS. Detergent effects of sodium deoxycholate are a major feature of an injectable phosphatidylcholine formulation used for localized fat dissolution. Dermatol Surg. 2004;30(7):1001-1008.

4. Kybella [package insert]. Westlake, California: Kythera Biopharmaceuticals; 2015.

5. Rzany B, Griffiths T, Walker P, Lippert S, McDiarmid J, Havlickova B. Reduction of unwanted submental fat with ATX-101 (deoxycholic acid), an adipocytolytic injectable treatment: results from a phase III, randomized, placebo-controlled study. $\mathrm{Br} J$ Dermatol. 2014;170(2):445-453.

6. Rotunda AM, Kolodney MS. Mesotherapy and phosphatidylcholine injections: historical clarification and review. Dermatol Surg. 2006;32(4):465-480.

7. Sobanko JF, Miller CJ, Alster TS. Topical anesthetics for dermatologic procedures: a review. Dermatol Surg. 2012;38(5):709-721.

8. Railan D, Alster TS. Use of topical lidocaine for cosmetic dermatologic procedures. J Drugs Dermatol. 2007;6(11):1104-1108. 